\title{
JUIN 1961
}

\section{REVUE}

\section{INTERNATIONA LE}

\author{
DE LA
}

\section{CROIX-ROUGE}

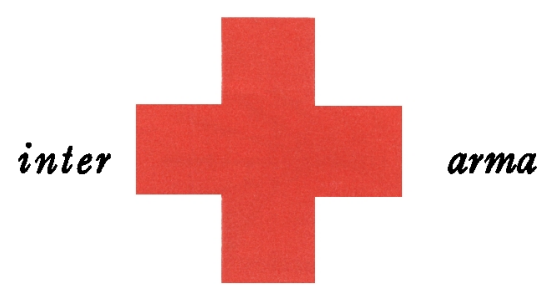

caritas 


\section{COMITÉ INTERNATIONAL DE LA CROIX-ROUGE}

\section{à Genève}

MM. LÉOPOLD BOISSIER, docteur en droit, professeur honoraire de l'Université de Genève, ancien s ecrétaire général de l'Union interparlementaire, président (1946) 1

JACQUES CHENEVIERE, docteur ès lettres h.c., vice-président d'honneur (1919)

M11e LUCIE ODIER, ex-chef du Service des infirmières-visiteuses de la Section genevoise de la Croix-Rouge suisse (1930)

MM. CARL J. BURCKHARDT, docteur en philosophie, ancien ministre de Suisse en France (1933)

MARTIN BODMER, docteur en philosophie h.c., vice-président (1940)

ERNEST GLOOR, docteur en médecine, vice-président (1945)

PAUL RUEGGER, ancien ministre de Suisse en Italie et en Grande-Bretagne, membre de la Cour Permanente d'Arbitrage (1948)

RODOLFO OLGIATI, docteur en médecine h.c., ancien directeur du Don suisse (1949)

M110 MARGUERITE VAN BERCHEM, ancien chef de service de l'Agence centrale des prisonniers de guerre (1851)

MM. FREDERIC SIORDET, avocat, conseiller du Comité international de la Croix-Rouge de 1943 à 1951, (1951)

MARCEL JUNOD, docteur en médecine, délégué du Comité international de la Croix-Rouge de 1935 à $1946(1952)$

GUILLAUME BORDIER, ingénieur dipl. E.P.F., M.B.A. Harvard, banquier (1955)

ADOLPHE FRANCESCHETTI, docteur en médecine, professeur de clinique ophtalmologique à l'Université de Genève (1958)

HANS BACHMANN, docteur en droit, secrétaire général adjoint du Comité international de la CroixRouge de 1944 a 1946 (1958)

JACQUES FREYMOND, docteur ès lettres, directeur de l'Institut universitaire de hautes études internationales, professeur à l'Université de Genève (1959)

DIETRICH SCHINDLER, docteur en droit (1961)

\section{Direction:}

MM. ROGER GALLOPIN, docteur en droit, directeur exécutif

JEAN S. PICTET, docteur en droit, directeur des Affaires générales

EDOUARD de BONDELI, sous-directeur, Services financiers et administratifs

CLAUDE PILLOUD, sous-directeur, Service juridique

1 Les années indiquées dans les parenthèses désignent les dates de nomination des membres du Comité international. 


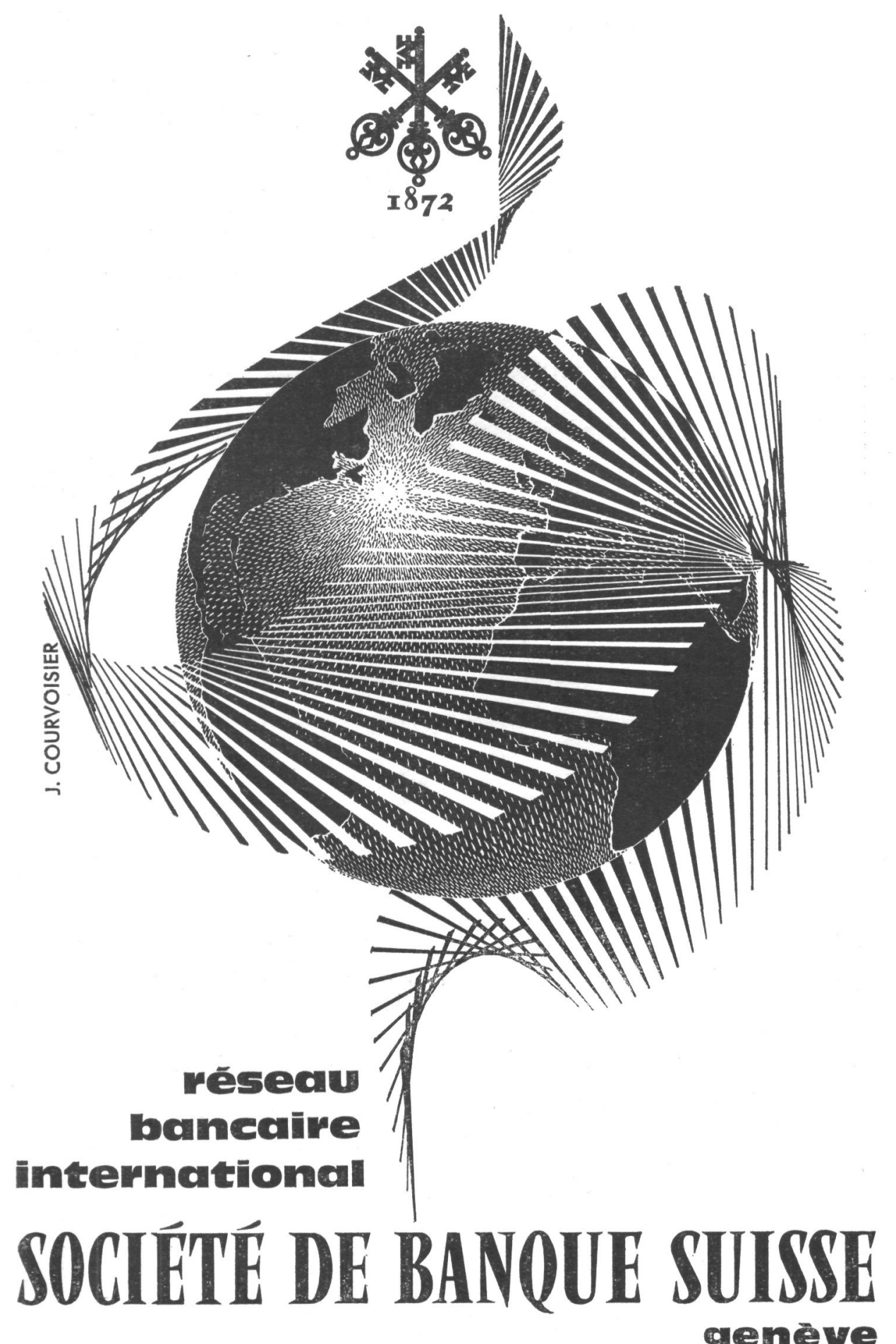




\section{SOCIETE FIDUCIAIRE ROMANDE OFOR S.A.}

9, rue d'Italie, Genève - Tél. 243293

— Expertises, revisions et organisations comptables

^ Domiciliation et administration de sociétés

$\downarrow$ Assainissements et liquidations

- Interventions et conseils en matière fiscale

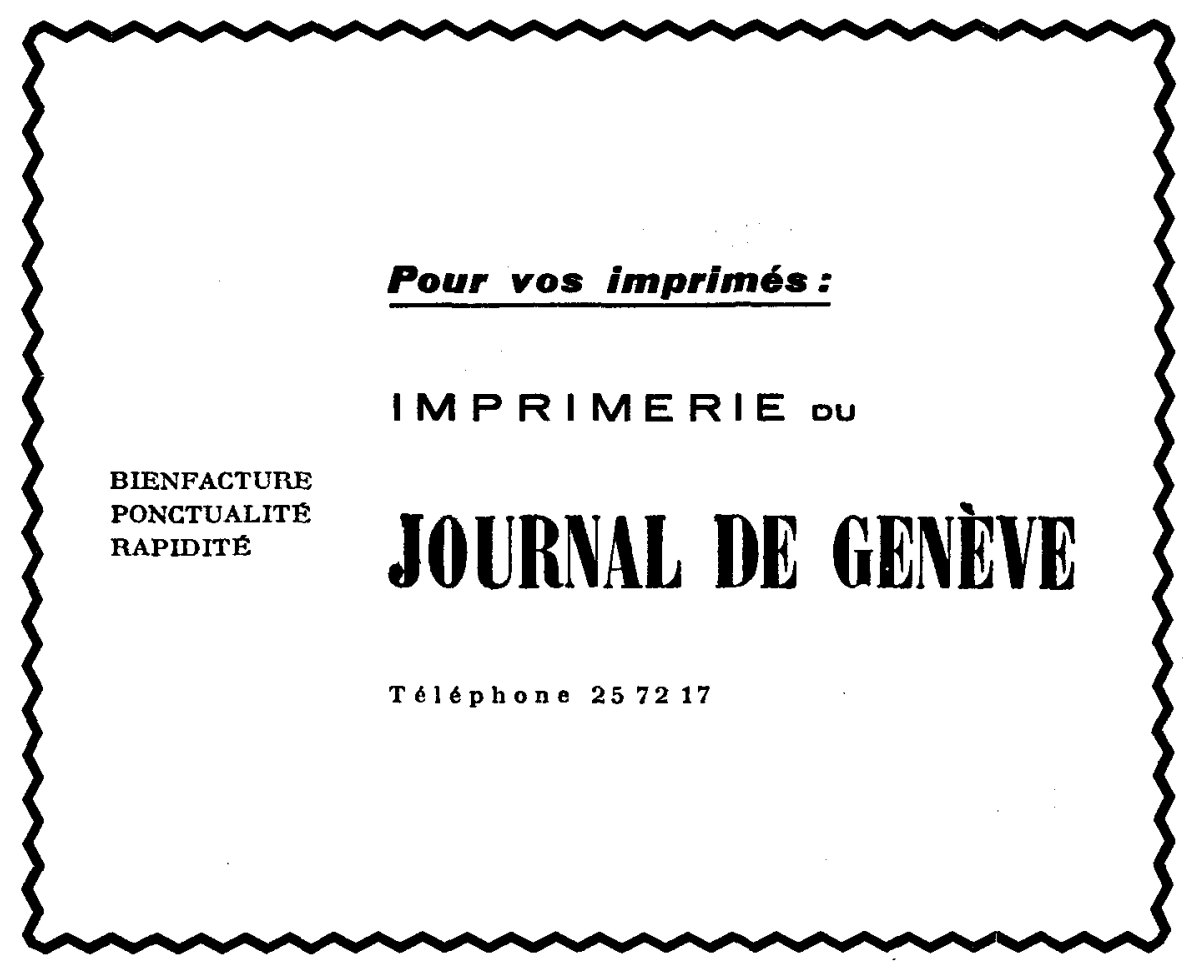




\title{
REVUE INTERNATIONALE \\ DE LA CROIX-ROUGE
}

\author{
QUARANTE-TROISIEME ANNEE - NO $510^{\circ}$ \\ JUIN 1961
}

SOMMAIRE

Pages

\section{COMITÉ INTERNATIONAL DE LA CROIX-ROUGE}

Reconnaissance de la Croix-Rouge du Nigéria (434 circulaire aux

Comilés centraux) . . . . . . . . . . . . . 265

Nouvelles d'ici et de partout. . . . . . . . . . . . . . 267

Le CICR visite des détenus au Congo. . . . . . . . . . . . . 274

Situation financière du CICR . . . . . . . . . . . . 275

Le Comité international et les événements de Cuba . . . . . . . 280

Voyage du Président du Comité international en Allemagne (Répu-

blique fédérale) .............. . $28 \mathbf{I}$

Pour le 8 mai .............. . . 283

\section{NOUVELLES DE SOCIÉTÉS NATIONALES}

Autriche . . . . . . . . . . . . . . . . . . . 285

Japon. . . . . . . . . . . . . . . . . . . . . 286

\section{CHRONIQUE}

Formation et emploi des aveugles dans les collectivités rurales. . . 292

\section{FAITS ET DOCUMENTS}

Une initiative humanitaire en France, à la fin du Premier Empire, par Julien LAmBERT . . . . . . . . . . . . 302 $8 \mathrm{e}$ entretien consacré au droit international médical . . . . . . 309

\section{A TRAVERS LES REVUES}




\section{ÉDITION ANGLAISE DE LA REVUE}

Une édition en langue anglaise paraît chaque mois sous le titre: International Review of the Red Cross

Elle est en principe identique à l'édition française, et peut être obtenue aux mêmes conditions.

\section{SUPPLEMENTS DE LA REVUE}

\section{EN LANGUE ESPAGNOLE}

Reconocimiento de la Cruz Roja de Nigeria (Circular Núm. 434).

Informaciones del mundo entero. - El CICR y los acontecimientos de Cuba. - El CICR visita a los detenidos en el Congo. - Viaje del Presidente del Comité Internacional a Alemania (República Federal).

\section{EN LANGUE ALLEMANDE}

Anerkennung des Roten Kreuzes von Nigeria (434. Rundschreiben). Nachrichten aus Nah und Fern. - Reise des Präsidenten des Internationalen Komitees nach der Bundesrepublik Deutschland. Das IKRK besucht Häftlinge im Kongo. - Das IKRK und die Ereignisse auf Kuba.

\section{LA}

\section{REVUE INTERNATIONALE DE LA CROIX-ROUGE} est publiée chaque mois par le Comité international de la Croix-Rouge

7, avenue de la Paix, Genève (Suisse) - Compte de chèques postaux I, 1767 Abonnement un an: Fr. 20,-: le numéro Fr. 2,-

Pour les pays suivants: Algérie, Allemagne, Autriche, Belgique, Danemark, Finlande, France, Italie, Luxembourg, Maroc, Norvège, Pays-Bas, Portugal, Sarre, Suedde, Tunisie, Vatican, on peut s'abonner auprès des bureaux de poste (fr. s. 20,50)

Rédaction : JEAN-G. LOSSIER 